\title{
Correction to: Major adverse cardiovascular events in non-valvular atrial fibrillation with chronic obstructive pulmonary disease: the ARAPACIS study
}

\author{
Valeria Raparelli ${ }^{1}$. Daniele Pastori ${ }^{1}$. Serena Francesca Pignataro ${ }^{1}$ - Anna Rita Vestri ${ }^{2}$. Pasquale Pignatelli ${ }^{1}$. \\ Roberto Cangemi ${ }^{1}$. Marco Proietti ${ }^{3}$. Giovanni Davi ${ }^{4}$. William Robert Hiatt ${ }^{5}$. Gregory Yoke Hong Lip ${ }^{3}$. \\ Gino Roberto Corazza ${ }^{6} \cdot$ Francesco Perticone $^{7} \cdot$ Francesco Violi $^{1} \cdot$ Stefania Basili ${ }^{1} \cdot$ ARAPACIS Study Collaborators
}

Published online: 3 August 2018

(c) Società Italiana di Medicina Interna 2018

\section{Correction to: \\ Internal and Emergency Medicine (2018) 13:651-660 \\ https://doi.org/10.1007/s11739-018-1835-9}

In the original publication, one of the ARAPACIS collaborators Dr. "Leonardo Di Gennaro" name has been erroneously mentioned as "Leonardo De Gennaro".

The original article can be found online at https://doi.org/10.1007/ s11739-018-1835-9.

Francesco Violi

francesco.violi@uniroma1.it

1 Department of Internal Medicine and Medical Specialties, Clinica Medica, Atherothrombosis Center, Sapienza University of Rome, Viale del Policlinico 155, 00161 Rome, Italy

2 Department of Public Health and Infections Disease, Sapienza University of Rome, Rome, Italy

3 University of Birmingham Institute of Cardiovascular Sciences, City Hospital, Birmingham, UK

4 Department of Medicine and Aging, University of Chieti "G. d'Annunzio, Chieti, Italy

5 Division of Cardiology, University of Colorado School of Medicine and CPC Clinical Research, Aurora, CO, USA

6 First Department of Internal Medicine, Fondazione IRCCS Policlinico San Matteo, University of Pavia, Pavia, Italy

7 Department of Medical and Surgical Sciences, University Magna Græcia of Catanzaro, Catanzaro, Italy 